\title{
Sausages Are Good Packings
}

\author{
J. Betke, ${ }^{1}$ M. Henk, ${ }^{2}$ and J. M. Wills ${ }^{1}$ \\ ${ }^{1}$ Math. Institut, Universität Siegen, \\ D-57068 Siegen, Germany \\ betke@mathematik.uni-siegen.d400.de \\ wills@hrz.uni-siegen.d400.de \\ ${ }^{2}$ Math. Institut, Technische Universität Berlin, \\ D-10623 Berlin, Germany \\ henk@math.tu-berlin.de
}

\begin{abstract}
Let $B^{d}$ be the $d$-dimensional unit ball and, for an integer $n$, let $C_{n}=\left\{x^{1}, \ldots, x^{n}\right\}$ be a packing set for $B^{d}$, i.e., $\left|x^{i}-x^{j}\right| \geq 2,1 \leq i<j \leq n$. We show that for every $\rho<\sqrt{2}$ a dimension $d(\rho)$ exists such that, for $d \geq d(\rho)$,
\end{abstract}

$$
V\left(\operatorname{conv}\left(C_{n}\right)+\rho B^{d}\right) \geq V\left(\operatorname{conv}\left(S_{n}\right)+\rho B^{d}\right),
$$

where $S_{n}$ is a "sausage" arrangement of $n$ balls, holds. This gives considerable improvement to Fejes Tóth's "sausage" conjecture in high dimensions. Further, we prove that, for every convex body $K$ and $\rho<\frac{1}{32} d^{-2}$,

$$
V\left(\operatorname{conv}\left(C_{n}\right)+\rho K\right) \geq V\left(\operatorname{conv}\left(S_{n}\right)+\rho K\right),
$$

where $C_{n}$ is a packing set with respect to $K$ and $S_{n}$ is a minimal "sausage" arrangement of $K$, holds.

\section{Introduction}

Throughout this paper $E^{d}$ denotes the $d$-dimensional Euclidean space equipped with the Euclidean norm $|\cdot|$ and scalar product $\langle\cdot, \cdot\rangle, B^{d}$ denotes the $d$-dimensional unit ball with boundary $S^{d-1}$ and $\operatorname{conv}(P)$ denotes the convex hull of a set $P \subset E^{d}$. The volume of $P$ with respect to the affine hull of $P$ is denoted by $V(P)$ and let $\kappa_{d}=V\left(B^{d}\right)$. Finally, the spherical volume is denoted by $V_{*}(\cdot)$.

The well-known density concept of infinite packings (see, e.g., [GL], [CS], and [FK]) has the inherent disadvantage that it does not work for finite packings. Further, the usual finite packings are always considered with respect to a given 
"large" convex body, e.g., a cube (a "bin" or "container"), into which the "small" convex bodies are packed (see, e.g., [Ro2], [GL], [FK], and [Gr2]). In contrast to these "bin packings" (for the name see, e.g., [KK] or [GW]), i.e., constrained finite packings, we are interested in "free packings", i.e., finite packings independent of a given "large" convex body.

First results for these free finite packings in the plane have been obtained by Thue [Th], Rogers [Ro1], Groemer [Gr1], and Oler [Ol]. A way to study the density of free packings for balls in higher dimensions was observed by L. Fejes Tóth [Fe]. To this end let $\mathscr{P}_{n}\left(B^{d}\right)$ be the set of all possible packing arrangements of $n$ translates of $B^{d}$ in $E^{d}$, i.e.,

$$
\mathscr{P}_{n}\left(B^{d}\right)=\left\{C_{n} \subset E^{d}: C_{n}=\left\{x^{1}, \ldots, x^{n}\right\},\left|x^{i}-x^{j}\right| \geq 2, i \neq j\right\} .
$$

Fejes Tóth suggested defining the density of a (finite) packing $C_{n} \in \mathscr{P}_{n}\left(B^{d}\right)$ of $n$ balls by

$$
\delta\left(B^{d}, C_{n}\right):=\frac{n \kappa_{d}}{V\left(\operatorname{conv}\left(C_{n}\right)+B^{d}\right)},
$$

and he asked to determine, for every $n$ and $d$,

$$
\delta\left(B^{d}, n\right)=\sup \left\{\delta\left(B^{d}, C_{n}\right): C_{n} \in \mathscr{P}_{n}\left(B^{d}\right)\right\} .
$$

In other words: What is the best possible arrangement of $n$ nonoverlapping $d$-dimensional unit balls with respect to the volume of the convex hull of the arrangement?

He observed that, at least for $d \geq 5$, the limit (with respect to $n$ ) of the densities of so-called "sausage" arrangements of balls are larger than the density of infinite packings. Here $S_{n}=\left\{x^{1}, \ldots, x^{n}\right\} \in \mathscr{P}_{n}\left(B^{d}\right)$ is called a "sausage" arrangement if $x^{i}=2 i \cdot u, 1 \leq i \leq n$, for some $u \in S^{d-1}$. More specifically he conjectured that, for $d \geq 5$, a "sausage" arrangement is a best possible arrangement:

"Sausage" Conjecture. For $d \geq 5$ and $n \in \mathbb{N}$,

$$
\delta\left(B^{d}, n\right)=\delta\left(B^{d}, S_{n}\right)
$$

holds.

For results related to this conjecture we refer to [GW]. Fejes Tóth's concept was generalized by Betke et al. [BHW] to parametric densities by defining, for every $\rho \geq 0$ and $C_{n} \in \mathscr{P}_{n}\left(B^{d}\right)$,

$$
\delta\left(B^{d}, C_{n}, \rho\right)=\frac{n \kappa_{d}}{V\left(\operatorname{conv}\left(C_{n}\right)+\rho B^{d}\right)}
$$

and

$$
\delta\left(B^{d}, n, \rho\right)=\sup \left\{\delta\left(B^{d}, C_{n}, \rho\right): C_{n} \in \mathscr{P}_{n}\left(B^{d}\right)\right\}
$$


These parametric densities are not only of interest for finite packings but they also connect the finite and the infinite packing problem. In fact is was shown in [BHW] that a small $\rho(\rho<2 / \sqrt{3})$ favors "sausage" arrangements while a large $\rho(\rho \geq 2)$ favors high-dimensional arrangements. Here we give an improvement of the bound $2 / \sqrt{3}$. More precisely we prove:

Theorem 1.1. For every $\rho<\sqrt{2}$ a dimension $d(\rho)$ exists such that for, $d \geq d(\rho)$ and $n \in \mathbb{N}$,

$$
\delta\left(B^{d}, n, \rho\right)=\delta\left(B^{d}, S_{n}, \rho\right)
$$

holds.

Since $\delta\left(B^{d}, n, \rho_{1}\right)=\delta\left(B^{d}, S_{n}, \rho_{1}\right)$ for a certain $\rho_{1}$ implies

$$
\delta\left(B^{d}, n, \rho\right)=\delta\left(B^{d}, S_{n}, \rho\right) \quad \text { for all } \quad \rho<\rho_{1}
$$

(see [BHW]), this theorem shows that in high dimensions the "sausage" arrangements are much better packings than required by Fejes Tóth's "sausage" conjecture.

Further, we may deduce from Theorem 1.1 the following upper bound for the density $\delta\left(B^{d}\right)$ of a densest infinite packing of balls (see Corollary 1.1 of [BHW]):

Corollary 1.1. For every $\varepsilon>0$ a $d(\varepsilon) \in \mathbb{N}$ exists such that, for $d \geq d(\varepsilon)$,

$$
\delta\left(B^{d}\right)<(\sqrt{2}-\varepsilon)^{-d} .
$$

Observe that this bound is asymptotically of the same order as the classical bounds due to Rogers [Ro2] and Blichfeldt [Bl]. Though this bound is much weaker than the best-known one (see, e.g., $[\mathrm{KL}]$ ) it shows that parametric densities also give a new approach to the study of infinite packings.

Now, the concept of parametric densities can easily be generalized to arbitrary convex bodies. To this end let $\mathscr{K}^{d}$ be the set of all convex bodies in $E^{d}$ with nonempty interior and let $\mathscr{K}_{0}^{d} \subset \mathscr{K}^{d}$ be the set of all centrally symmetric convex bodies. The distance function of $K \in \mathscr{K}_{0}^{d}$ is denoted by $f_{K}$, i.e.,

$$
f_{K}(x)=\min \{\lambda \geq 0: x \in \lambda K\}
$$

Further, for $K \in \mathscr{K}^{d}$ let $\mathrm{D} K \in \mathscr{K}_{0}^{d}$ be the difference body, i.e., $\mathrm{D} K=\frac{1}{2}(K-K)$. For $n \in \mathbb{N}, K \in \mathscr{K}^{d}$ let $\mathscr{P}_{n}(K)$ be the set of all possible packing arrangements of $n$ translates of $K$, which can be defined by

$$
\mathscr{P}_{n}(K)=\left\{C_{n} \subset E^{d}: C_{n}=\left\{x^{1}, \ldots, x^{n}\right\}, x^{i}+\operatorname{int}(K) \cap x^{j}+\operatorname{int}(K)=\varnothing, i \neq j\right\},
$$

where int $(\cdot)$ denotes the interior. Since

$$
C_{n} \in \mathscr{P}_{n}(K) \quad \Leftrightarrow \quad C_{n} \in \mathscr{P}_{n}(\mathrm{D} K),
$$


we may write $\mathscr{P}_{n}(K)$ as

$$
\mathscr{P}_{n}(K)=\left\{C_{n} \subset E^{d}: C_{n}=\left\{x^{1}, \ldots, x^{n}\right\}, f_{\mathrm{D} K}\left(x^{i}-x^{j}\right) \geq 2, i \neq j\right\}
$$

As in the case of the ball we consider the following parametric densities for $K \in \mathscr{K}^{d}$ :

$$
\begin{aligned}
\delta\left(K, C_{n}, \rho\right) & =\frac{n \cdot V(K)}{V\left(\operatorname{conv}\left(C_{n}\right)+\rho K\right)} \\
\delta(K, n, \rho) & =\sup \left\{\delta\left(K, C_{n}, \rho\right): C_{n} \in \mathscr{P}_{n}(K)\right\}
\end{aligned}
$$

In [BHW] it was shown that for $K \in \mathscr{K}_{0}^{d}$ a positive constant $\rho_{m}$ exists depending only on the dimension such that, for $\rho<\rho_{m}, \delta(K, n, \rho)$ is attained for a certain "sausage" arrangement, where for $u \in S^{d-1}$ the set $S_{n}(u, K)=\left\{x^{1}, \ldots, x^{n}\right\} \in \mathscr{P}_{n}(K)$ is called a "sausage" arrangement in direction $u$ iff $x^{i}=2 i \cdot u / f_{\mathrm{DK}}(u)$ for $1 \leq i \leq n$. Here we give an explicit bound for such a $\rho_{m}$ for arbitrary convex bodies. To this end we denote by $r(K)$ and $R(K)$ the inradius and circumradius of a convex body.

Theorem 1.2. Let $K \in \mathscr{K}^{d}, n \in \mathbb{N}$, and $\rho \leq \frac{1}{32} d^{-1} r(K) / R(K)$. Then

$$
\delta(K, n, \rho)=\max _{u \in S^{d-1}} \delta\left(K, S_{n}(u, K), \rho\right)
$$

By John's [Jo] theorem we can always achieve, by an affine transformation $A$, that the ratio $r(A K) / R(A K)$ is bounded from below by $d^{-1}$ for $K \in \mathscr{K}^{d}$. For $K \in \mathscr{K}_{0}^{d}$ even $r(A K) / R(A K) \geq d^{-1 / 2}$ holds. Since $\delta\left(K, C_{n}, \rho\right)$ is invariant under affine mappings (see [BHW]) we can deduce, from Theorem 1.2:

Corollary 1.2. Let $K \in \mathscr{K}^{d}, n \in \mathbb{N}$, and $\rho \leq \frac{1}{32} d^{-2}$. Then

$$
\delta(K, n, \rho)=\max _{u \in S^{d-1}} \delta\left(K, S_{n}(u, K), \rho\right)
$$

Corollary 1.3. Let $K \in \mathscr{K}_{0}^{d}, n \in \mathbb{N}$, and $\rho \leq \frac{1}{32} d^{-3 / 2}$. Then

$$
\delta(K, n, \rho)=\max _{u \in S^{d-1}} \delta\left(K, S_{n}(u, K), \rho\right)
$$

Let us remark that for centrally symmetric convex bodies in the plane the exact bound is $\frac{3}{4}$. Moreover, for $K \in \mathscr{K}_{0}^{d}\left(K \in \mathscr{K}^{d}\right)$ and for $\rho \geq 2(\rho \geq d+1)$, $\lim \sup \delta(K, n, \rho)$ converges to the density of the densest infinite packing as $n$ tends to infinity [BHW]. 


\section{Proof of Theorem 1.1}

As mentioned in the Introduction the following weaker version of Theorem 1.1 was proved in [BHW]:

Theorem. For every $\rho<2 / \sqrt{3}$ a dimension $d(\rho)$ exists such that, for $d \geq d(\rho)$ and $n \in \mathbb{N}$,

$$
\delta\left(B^{d}, n, \rho\right)=\delta\left(B^{d}, S_{n}, \rho\right)
$$

holds.

To describe the main tool used for the proof of the theorem we need some more notation. For an arrangement $C_{n} \in \mathscr{P}_{n}\left(B^{d}\right)$ and $x^{j} \in C_{n}$ let

$$
H_{j}=\left\{x \in E^{d}:\left|x-x^{j}\right| \leq\left|x-x^{i}\right| \text { for all } x^{i} \in C_{n}\right\}
$$

denote the Dirichlet-Voronoï cell of $x^{j}$ with respect to the arrangement. Further, for a $(d-2)$-dimensional linear subspace $L \subset E^{d}$ and for $\rho>0$ let

$$
\begin{aligned}
& M_{j}(\rho, L)=\left\{z \in S^{d-1} \cap L: x^{j}+\rho z \notin H_{j}\right\}, \\
& K_{j}(\rho, L)=\left\{z \in S^{d-1} \cap L: x^{j}+\rho z \in H_{j}\right\} .
\end{aligned}
$$

In [BHW] the following remark was made at the end of the proof of Theorem 4.1:

Remark. Let $\rho \in \mathbb{R}^{>0}$ have the following property: a $d_{0} \in \mathbb{N}$ and a $c \in \mathbb{R}$ exist such that, for all $d \geq d_{0}, n \in \mathbb{N}$, and $C_{n} \in \mathscr{P}_{n}\left(B^{d}\right)$,

$$
V_{*}\left(K_{j}(\rho, L)\right) \geq c \cdot V_{*}\left(S^{d-2}\right)
$$

holds for all ( $d-2$ )-dimensional linear subspaces $L$ and for every Dirichlet-Voronoï cell $H_{j}$ of $C_{n}$. Then a $d(\rho) \in \mathbb{N}$ exists such that $\delta\left(B^{d}, n, \rho\right)=\delta\left(B^{d}, S_{n}, \rho\right)$ for all $d \geq d(\rho)$ and $n \in \mathbb{N}$.

In [BHW] this was proved for every $\rho<2 / \sqrt{3}$ which implies the above theorem. Here we use Rogers' [Ro2] technique for the estimation of the size of the Dirichlet-Voronoï cells of packings to find:

Theorem 2.1. Let $0<\rho<\sqrt{2}$. Then there is a constant $c>0$ and $a d_{0} \in \mathbb{N}$ such that, for all $n \in \mathbb{N}$ and every packing $C_{n} \in \mathscr{P}_{n}\left(B^{d}\right)$ with $d \geq d_{0}$, it holds that, for every $x^{j} \in C_{n}$ and every $(d-2)$-dimensional linear subspace $L$,

$$
\frac{V_{*}\left(M_{j}(\rho, L)\right)}{V_{*}\left(K_{j}(\rho, L)\right)} \leq c .
$$


Since $V_{*}\left(M_{j}(\rho, L)\right)+V_{*}\left(K_{j}(\rho, L)\right)=V_{*}\left(S^{d-2}\right)$ we obtain Theorem 1.1 as an immediate consequence of the above remark.

In the course of our investigation we need to integrate over a part of a simplex. This can be done most conveniently by using the following "simplicial coordinates":

Lemma 2.1. Let $S \subset E^{d}$ be a $d$-simplex, let $F_{k}$ be a $k$-face of $S, k \leq d-1$, and let $\bar{F}_{k}$ be the $(d-k-1)$-face of $S$ with $F_{k} \cap \bar{F}_{k}=\varnothing$. For a measurable subset $G \subset S$ ana' $a$ continuous function $f$ on $S$ we have

$$
\begin{aligned}
\int_{G} f d x= & \frac{d !}{k !(d-1-k) !} \frac{V(S)}{V\left(F_{k}\right) V\left(\bar{F}_{k}\right)} \\
& \cdot \int_{F_{k}} \int_{\bar{F}_{k}} \int_{u \bar{x}+(1-\mu)} f(\mu \bar{x}+(1-\mu) x) \mu^{d-1-k}(1-\mu)^{k} d \mu d \bar{x} d x .
\end{aligned}
$$

Remark. Here the notation $\int d x$ means integration in a space of appropriate dimension.

Proof. To compute the integral let $S=\operatorname{conv}\left\{a^{0}, \ldots, a^{d}\right\}, F_{k}=\operatorname{conv}\left\{a^{0}, \ldots, a^{k}\right\}$, and $\bar{F}_{k}=\operatorname{conv}\left\{a^{k+1}, \ldots, a^{d}\right\}$. Further, let $A, A_{k}$, and $\bar{A}_{k}$ denote the matrices

$$
\begin{gathered}
A=\left(a^{1}-a^{0}, \ldots, a^{d}-a^{0}\right), \quad A_{k}=\left(a^{1}-a^{0}, \ldots, a^{k}-a^{0}\right), \text { and } \\
\overline{A_{k}}=\left(a^{k+2}-a^{k+1}, \ldots, a^{d}-a^{k+1}\right) .
\end{gathered}
$$

Finally, we denote by $T^{d}$ the standard simplex

$$
T^{d}=\left\{\left(x_{1}, \ldots, x_{d}\right)^{T} \in E^{d}: x_{i} \geq 0, \sum_{i=1}^{d} x_{i} \leq 1\right\}
$$

Now we apply a number of transformations. First let $\varphi_{1}: E^{d} \rightarrow E^{d}$ be given by $\varphi_{1}(x)=A x+a^{0}$. Then $T^{d}=\varphi_{1}^{-1}(S)$ and thus

$$
\int_{G} f(x) d x=d ! V(S) \int_{\varphi_{1}^{-1}(G)} f\left(\varphi_{1}(x)\right) d x
$$

Next we consider $\varphi_{2}: E^{k} \times \mathrm{E} \times \mathrm{E}^{d-1-k} \rightarrow E^{d}$ with

$$
\begin{aligned}
& \varphi_{2}\left(\mu_{1}, \ldots, \mu_{k}, \mu, \mu_{k+2}, \ldots, \mu_{d}\right) \\
& =(1-\mu) \cdot\left(\mu_{1}, \ldots, \mu_{k}, 0, \ldots, 0\right)^{T} \\
& \quad+\mu \cdot\left(0, \ldots, 0,1-\sum_{i=k+2}^{d} \mu_{i}, \mu_{k+2}, \ldots, \mu_{d}\right)^{T} .
\end{aligned}
$$

$\varphi_{2}$ is a bijection from the interior of $T^{k} \times T^{1} \times T^{d-1-k}$ onto the interior of $T^{d}$. By a straightforward calculation we find, for the determinant of the Jacobian $D \varphi_{2}$ of 
$\varphi_{2}, \operatorname{det}\left(D \varphi_{2}\right)=\mu^{d-1-k}(1-\mu)^{k}$. Thus with $\mu^{\prime}=\left(\mu_{1}, \ldots, \mu_{k}\right), \bar{\mu}=\left(\mu_{k+2}, \ldots, \mu_{d}\right)$, and (2.1),

$$
\begin{aligned}
\int_{G} f(x) d x= & ! V(S) \int_{T^{k}} \int_{T^{d-1-k}} \int_{\varphi_{2}\left(\mu^{\prime}, \mu, \bar{\mu}\right) \in \varphi_{1}^{-1}(G)} f\left(\varphi_{1}\left(\varphi_{2}\left(\mu^{\prime}, \mu, \bar{\mu}\right)\right)\right) \\
& \times \mu^{d-1-k}(1-\mu)^{k} d \mu d \bar{\mu} d \mu^{\prime}
\end{aligned}
$$

As

$$
\begin{gathered}
\varphi_{1}\left(\varphi_{2}\left(\mu^{\prime}, \mu, \bar{\mu}\right)\right)=(1-\mu)\left(A_{k} \mu^{\prime}+a^{0}\right)+\mu\left(\overline{A_{k}} \bar{\mu}+a^{k+1}\right), \\
F_{k}=\left\{A_{k} \mu^{\prime}+a^{0}: \mu^{\prime} \in T^{k}\right\}
\end{gathered}
$$

and

$$
\bar{F}_{k}=\left\{\overline{A_{k}} \bar{\mu}+a^{k+1}: \bar{\mu} \in T^{d-1-k}\right\}
$$

we obtain the required formula.

Further, we need the quotient of two certain integrals to be bounded:

Lemma 2.2. Let $k, \bar{k} \in \mathbb{N}$ with $\bar{k} \geq k+1$ and let $\alpha, \beta, \gamma \in \mathbb{R}$ with $\gamma>\beta>0$, $\alpha>0$. Then for $a, b, c \in \mathbb{R}, d \in \mathbb{N}$ with $b, c \geq 0, b<c, a \geq \alpha, a^{2}+c^{2} \geq \gamma, a^{2}+$ $b^{2} \leq \beta, d \geq \bar{k}$, the quotient

$$
\frac{\int_{0}^{\mu_{0}}\left(\sqrt{a^{2}+(\mu c+(1-\mu) b)^{2}}\right)^{-(d+1)} \mu^{d-1-k}(1-\mu)^{k} d \mu}{\int_{\mu_{0}}^{1}\left(\sqrt{a^{2}+(\mu c+(1-\mu) b)^{2}}\right)^{-(d+1)} \mu^{d-1-k}(1-\mu)^{k} d \mu}
$$

where $\mu_{0} \in[0,1]$ is determined by $a^{2}+\left(\mu_{0} c+\left(1-\mu_{0}\right) b\right)^{2}=\beta$, is maximal for $a=\alpha, b=0, a^{2}+c^{2}=\gamma$, and $d=\bar{k}$.

Proof. First we show that the quotient is monotonely decreasing in $a, b, c, d$. To do this we set

$$
f(a, b, c, d, \mu)=\left(\sqrt{a^{2}+(\mu c+(1-\mu) b)^{2}}\right)^{-(d+1)} \mu^{d-1-k}(1-\mu)^{k} .
$$

For $d$ we have, by the mean value theorem of integral calculus,

$$
\begin{gathered}
\frac{\int_{0}^{\mu_{0}} f(a, b, c, d+1, \mu) d \mu}{\int_{\mu_{0}}^{1} f(a, b, c, d+1, \mu) d \mu}=\frac{\mu_{1} / \sqrt{a^{2}+\left(\mu_{1} c+\left(1-\mu_{1}\right) b\right)^{2}}}{\mu_{2} / \sqrt{a^{2}+\left(\mu_{2} c+\left(1-\mu_{2}\right) b\right)^{2}}} \\
\frac{\int_{0}^{\mu_{0}} f(a, b, c, d, \mu) d \mu}{\int_{\mu_{0}}^{1} f(a, b, c, d, \mu) d \mu}
\end{gathered}
$$


for suitable

$$
0 \leq \mu_{1} \leq \mu_{0} \leq \mu_{2} \leq 1 .
$$

Since the function $\mu / \sqrt{a^{2}+(\mu c+(1-\mu) b)^{2}}$ is monotonely increasing in $\mu$ we get that (2.2) is monotonely decreasing in $d$.

Now let $c^{\prime}>c$ and let $\mu_{0}^{\prime} \in[0,1]$ be given by $a^{2}+\left(\mu_{0}^{\prime} c^{\prime}+\left(1-\mu_{0}^{\prime}\right) b\right)^{2}=\beta$. We apply the linear transformation $\mu=(c-b) /\left(c^{\prime}-b\right) \mu^{\prime}$ and obtain

$$
\begin{aligned}
& \frac{\int_{0}^{\mu_{0}^{\prime}} f\left(a, b, c^{\prime}, d, \mu\right) d \mu}{\int_{u_{0}^{\prime}}^{1} f\left(a, b, c^{\prime}, d, \mu\right) d \mu} \\
& \quad=\frac{\left(\frac{c-b}{c^{\prime}-b}\right)^{d-k} \int_{0}^{\mu_{0}} f\left(a, b, c, d, \mu^{\prime}\right)\left(\frac{1-(c-b) /\left(c^{\prime}-b\right) \mu^{\prime}}{1-\mu^{\prime}}\right)^{k} d \mu^{\prime}}{\left(\frac{c-b}{c^{\prime}-b}\right)^{d-k} \int_{\mu_{0}}^{\left(c^{\prime}-b\right) /(c-b)} f\left(a, b, c, d, \mu^{\prime}\right)\left(\frac{1-(c-b) /\left(c^{\prime}-b\right) \mu^{\prime}}{1-\mu^{\prime}}\right)^{k} d \mu^{\prime}} .
\end{aligned}
$$

As $\left(c^{\prime}-b\right) /(c-b)>1$ and $\left(1-(c-b) /\left(c^{\prime}-b\right) \mu^{\prime}\right) /\left(1-\mu^{\prime}\right)$ is monotonely increasing for $0 \leq \mu^{\prime} \leq 1$ we find, again from the mean value theorem of integral calculus,

$$
\frac{\int_{0}^{\mu_{0}^{\prime}} f\left(a, b, c^{\prime}, d, \mu\right) d \mu}{\int_{\mu_{0}^{\prime}}^{1} f\left(a, b, c^{\prime}, d, \mu\right) d \mu} \leq \frac{\int_{0}^{\mu_{0}} f\left(a, b, c, d, \mu^{\prime}\right) d \mu^{\prime}}{\int_{\mu_{0}}^{1} f\left(a, b, c, d, \mu^{\prime}\right) d \mu^{\prime}} .
$$

Quite analoguously we find that (2.2) is monotonely decreasing in $b$.

Finally, let $a^{\prime}>a$. For the corresponding value $\mu_{0}^{\prime}$ we have $\mu_{0}^{\prime}<\mu_{0}$ and further we may write $a^{\prime 2}+(\mu c+(1-\mu) b)^{2}=g(\mu)\left(a^{2}+(\mu c+(1-\mu) b)^{2}\right)$ for an appropriate function $g$. We easily check that $g$ is monotonely decreasing in $\mu$ for $\mu \geq 0$. Thus we find, as before,

$$
\frac{\int_{0}^{\mu_{0}^{\prime}} f\left(a^{\prime}, b, c, d, \mu\right) d \mu}{\int_{\mu_{0}^{\prime}}^{1} f\left(a^{\prime}, b, c, d, \mu\right) d \mu}<\frac{\int_{0}^{\mu_{0}} f\left(a^{\prime}, b, c, d, \mu\right) d \mu}{\int_{\mu_{0}}^{1} f\left(a^{\prime}, b, c, d, \mu\right) d \mu} \leq \frac{\int_{0}^{\mu_{0}} f(a, b, c, d, \mu) d \mu}{\int_{\mu_{0}}^{1} f(a, b, c, d, \mu) d \mu} .
$$

Hence we may assume $b=0$ and $c^{2}=\gamma-a^{2}$. So $\mu_{0}=\sqrt{\left(\beta-a^{2}\right) /\left(\gamma-a^{2}\right)}$ and we see that $\mu_{0}$, regarded as a function in $a$, is monotonely decreasing in $a$. Now let $a^{\prime}>a, c^{\prime}=\gamma-\left(a^{\prime}\right)^{2}$, and $\mu_{0}^{\prime}$ be the value of $\mu$ with respect to $a^{\prime}$ and $c^{\prime}$. The function $g(\mu)$ defined by $a^{\prime 2}+\left(\mu c^{\prime}\right)^{2}=g(\mu)\left(a^{2}+(\mu c)^{2}\right)$ is monotonely decreasing in $\mu$ and as above we find

$$
\frac{\int_{0}^{\mu_{0}^{\prime}} f\left(a^{\prime}, 0, c^{\prime}, d, \mu\right) d \mu}{\int_{\mu_{0}^{\prime}}^{1} f\left(a^{\prime}, 0, c^{\prime}, d, \mu\right) d \mu}<\frac{\int_{0}^{\mu_{0}} f\left(a^{\prime}, 0, c^{\prime}, d, \mu\right) d \mu}{\int_{\mu_{0}}^{1} f\left(a^{\prime}, 0, c^{\prime}, d, \mu\right) d \mu} \leq \frac{\int_{T_{0}}^{\mu_{0}} f(a, 0, c, d, \mu) d \mu}{\int_{\mu_{0}}^{1} f(a, 0, c, d, \mu) d \mu} .
$$


Hence the integral in (2.2) is increased if $a$ is decreased and $a^{2}+c^{2}$ is kept constant. Thus the lemma is proved.

Now we come to the proof of Theorem 2.1.

Proof of Theorem 2.1. Let $\rho<\sqrt{2}, d_{0} \in \mathbb{N}$ with $d_{0}>\max \left(4,\left(4-\rho^{2}\right) /\left(2-\rho^{2}\right)\right\}$ and let $n \in \mathbb{N}, d \geq d_{0}$, and $C_{n} \in \mathscr{P}_{n}\left(B^{d}\right)$. Further, let $H$ be an arbitrary Dirichlet-Voronoi cell of the arrangement with respect to $x^{1}$, say, and let $L$ be a $(d-2)$-dimensional linear subspace. Obviously we may assume $x^{1}=0$. In the following we show that a constant $c \in \mathbb{R}$ exists such that

$$
\frac{V_{*}\left(M_{1}(\rho, L)\right)}{V_{*}\left(K_{1}(\rho, L)\right)} \leq c .
$$

To this end we consider the bounded polyhedron $H \cap W^{d}$, where $W^{d}$ denotes the $d$-cube with midpoint 0 and inradius $\sqrt{2}$. Observe that

$$
\begin{aligned}
& M_{1}(\rho, L)=\left\{z \in S^{d-1} \cap L: \rho z \notin H \cap W^{d}\right\}, \\
& K_{1}(\rho, L)=\left\{z \in S^{d-1} \cap L: \rho z \in H \cap W^{d}\right\} .
\end{aligned}
$$

Now we use a technique due to Rogers [Ro2] which gives, by an inductive construction, a dissection of the intersection $\left(H \cap W^{d}\right) \cap L$ into simplices $S$ of the form $S=\operatorname{conv}\left\{c^{0}, \ldots, c^{d-2}\right\}$, such that each $c^{i}$ is contained in a $(d-2-i)$-face $F$ of $\left(H \cap W^{d}\right) \cap L$ which contains conv $\left\{c^{i}, \ldots, c^{d-2}\right\}$ and is the nearest point of $F$ to 0 .

Let $c^{0}=0$ and assume we have chosen a sequence of points $\left\{c^{0}, \ldots, c^{i}\right\}$ such that $c^{j}$ lies in a $(d-2-j)$-face $F_{d-2-j}$ of $\left(H \cap W^{d}\right) \cap L$ and is the nearest point of the face to the origin, $0 \leq j \leq i$. Let $F_{d-3-i}^{1}, \ldots, F_{d-3-i}^{k}$ be the facets of $F_{d-2-i}$ which do not contain $c^{i}$. Then for $1 \leq j \leq k$ we define the point $c^{i+1, j}$ to be the point in $F_{d-3-i}^{j}$ nearest to 0 . So we obtain sequences $\left\{c^{0}, \ldots, c^{i}, c^{i+1, j}\right\}, 0 \leq j \leq k$, and at the end of the construction ( $i=d-2$ ) we obtain a dissection of the desired form.

If $c^{i}$ belongs to a face of $W^{d}$, then we clearly have $\left|c^{i}\right| \geq \sqrt{2}$. Otherwise, if $c^{i}$ lies in a $(d-2-i)$-face of $H$ we have, by a result of Rogers [Ro2], $\left|c^{i}\right| \geq \sqrt{2} \sqrt{i /(i+1)}$. Thus we get, for $0 \leq i \leq d-2$,

$$
\left|c^{i}\right| \geq \sqrt{2} \sqrt{\frac{i}{i+1}} .
$$

Let $S=\operatorname{conv}\left\{c^{0}, \ldots, c^{d-2}\right\}$ be an arbitrary but fixed simplex of this dissection, let $C$ be the cone generated by $c^{1}, \ldots, c^{d-2}$, and let

$$
M=\left\{z \in\left(S^{d-1} \cap L\right) \cap C: \rho z \notin S\right\}, \quad K=\left\{z \in\left(S^{d-1} \cap L\right) \cap C: \rho z \in S\right\} .
$$

To show (2.3) it is clearly sufficient to show that $V_{*}(M) / V_{*}(K)$ is bounded from above. In order to evaluate these spherical volumes we apply several transformations. First we replace the sets $M, K$ by the associated parts $M_{B}, K_{B}$ of the $d$-ball, i.e.,

$$
M_{B}=\left\{z \in\left(B^{d} \cap L\right) \cap C: \frac{\rho z}{|z|} \notin S\right\}, \quad K_{B}=\left\{z \in\left(B^{d} \cap L\right) \cap C: \frac{\rho z}{|z|} \in S\right\} .
$$


Clearly, we have $V_{*}(M) / V_{*}(K)=V\left(M_{B}\right) / V\left(K_{B}\right)$. In the next step we express $V\left(M_{B}\right), V\left(K_{B}\right)$ as integrals over the simplex $S$. To this end let $v \in S^{d-1} \cap L$ be the outward unit normal vector of the facet $F=\operatorname{conv}\left\{c^{1}, \ldots, c^{d-2}\right\}$ with respect to $S$ and let $\eta \in \mathbb{R}$ be the distance of the affine hull of $F$ to the origin. We consider the map $\varphi: E^{d} \cap L \rightarrow E^{d} \cap L$ given by $\varphi(x)=(\langle v, x\rangle /(\eta|x|)) x$. For the determinant of the Jacobian we find $\operatorname{det}(D \varphi)=(\langle v, x\rangle /(\eta|x|))^{d-2}$ and with

$$
M_{S}=\varphi^{-1}\left(M_{B}\right)=\left\{z \in S: \frac{\rho z}{|z|} \notin S\right\}, \quad K_{S}=\varphi^{-1}\left(K_{B}\right)=\left\{z \in S: \frac{\rho z}{|z|} \in S\right\}
$$

we obtain

$$
V\left(M_{B}\right)=\int_{M_{S}}\left(\frac{\langle v, x\rangle}{\eta|x|}\right)^{d-2} d x, \quad V\left(K_{B}\right)=\int_{K_{S}}\left(\frac{\langle v, x\rangle}{\eta|x|}\right)^{d-2} d x
$$

Now we use Lemma 2.1 with $k=0, F_{0}=0$, and $\bar{F}_{0}=F$. As $\langle v, x\rangle /(\eta|x|)$ is constant on rays emanating from 0 we get

$$
V\left(M_{B}\right)=\frac{\eta}{d-2} \int_{M_{F}}\left(\frac{1}{|x|}\right)^{d-2} d x, \quad V\left(K_{B}\right)=\frac{\eta}{d-2} \int_{K_{F}}\left(\frac{1}{|x|}\right)^{d-2} d x
$$

with $M_{F}=\{z \in F:|z| \leq \rho\}$ and $K_{F}=\{z \in F:|z| \geq \rho\}$.

Finally, by the definition of $d_{0}$ we may choose a $k \in \mathbb{N}, d-4 \geq k$, such that $\sqrt{2} \sqrt{(k+2) /(k+3)}>\rho$ (see (2.4)). We apply Lemma 2.1 to the $(d-3)$-simplex $F$ and the faces $F_{k}=\operatorname{conv}\left\{c^{1}, \ldots, c^{k+1}\right\}, \bar{F}_{k}=\operatorname{conv}\left\{c^{k+2}, \ldots, c^{d-2}\right\}$. Defining

$$
\xi\left(F, F_{k-1}\right)=\frac{\eta}{d-2} \frac{(d-3) !}{k !(d-4-k) !} \frac{V(F)}{V\left(F_{k}\right) V\left(\bar{F}_{k}\right)}
$$

we may write, by $(2.5)$,

$$
\begin{aligned}
& V\left(M_{B}\right)=\xi\left(F, F_{k}\right) \int_{F_{k}} \int_{\bar{F}_{k}} \int_{|\mu \bar{x}+(1-\mu) x| \leq \rho} \frac{\mu^{d-4-k}(1-\mu)^{k}}{|\mu \bar{x}+(1-\mu) x|^{d-2}} d \mu d \bar{x} d x, \\
& V\left(K_{B}\right)=\xi\left(F, F_{k}\right) \int_{F_{k}} \int_{\bar{F}_{k}} \int_{|\mu \bar{x}+(1-\mu) x| \geq \rho} \frac{\mu^{d-4-k}(1-\mu)^{k}}{|\mu \bar{x}+(1-\mu) x|^{d-2}} d \mu d \bar{x} d x .
\end{aligned}
$$

Thus to prove that $V_{*}(M) / V_{*}(K)$ is bounded from above it is sufficient to show

$$
\frac{\int_{|\mu \bar{x}+(1-\mu) x| \leq \rho}|\mu \bar{x}+(1-\mu) x|^{-(d-2)} \mu^{d-4-k}(1-\mu)^{k} d \mu}{\int_{|u \bar{x}+(1-\mu) x| \geq \rho}|\mu \bar{x}+(1-\mu) x|^{-(d-2)} \mu^{d-4-k}(1-\mu)^{k} d \mu} \leq \tilde{c}
$$

for all $x \in F_{k}, \bar{x} \in \bar{F}_{k}$, and a suitable constant $\tilde{c}$. 
By construction of $S$ we have that $\mid \mu \bar{x}+(1-\mu) x) \mid$ is strictly increasing in $\mu$. Further, we have $|x| \geq 1$ and, by the choice of $k,|\bar{x}|>\rho$ (see (2.4)). Thus for $|x| \geq \rho$ there is nothing to prove. Otherwise we find that the inequality (2.6) is of the form

$$
\frac{\int_{0}^{\mu_{0}}\left(\sqrt{a^{2}+(\mu c+(1-\mu) b)^{2}}\right)^{-(d-2)} \mu^{d-4-k}(1-\mu)^{k} d \mu}{\int_{\mu_{0}}^{1}\left(\sqrt{a^{2}+(\mu c+(1-\mu) b)^{2}}\right)^{-(d-2)} \mu^{d-4-k}(1-\mu)^{k} d \mu},
$$

where $a \geq \alpha=1$ denotes the distance of the line through $\bar{x}, x$ from $0, b$ is given by $a^{2}+b^{2}=|x|^{2}, c$ by $a^{2}+c^{2}=|\bar{x}|^{2}$, and $\mu_{0}$ is determined by

$$
a^{2}+\left(\mu_{0} c+\left(1-\mu_{0}\right) b\right)^{2}=\rho^{2}
$$

However, now (2.6) follows from Lemma 2.2 with $\beta=\rho^{2}$ and

$$
\gamma=\sqrt{2} \sqrt{(k+2) /(k+3)}
$$

and thus Theorem 2.1 is proved.

\section{Proof of Theorem $\mathbf{1 . 2}$}

From the definition it follows that the calculation of $\delta\left(K, C_{n}, \rho\right)$ for $K \in \mathscr{K}^{d}$ and $C_{n} \in \mathscr{P}_{n}(K)$ requires information on $V\left(\operatorname{conv}\left(C_{n}\right)+\rho K\right)$, which can be written as a polynomial in $\rho$ with the mixed volumes $V_{i}\left(\operatorname{conv}\left(C_{n}\right), K\right)$ (see [Sc]) as coefficients:

$$
V\left(\operatorname{conv}\left(C_{n}\right)+\rho K\right)=\sum_{i=0}^{d}\left(\begin{array}{l}
d \\
i
\end{array}\right) \rho^{d-i} V_{i}\left(\operatorname{conv}\left(C_{n}\right), K\right)
$$

In particular $V_{d}\left(\operatorname{conv}\left(C_{n}\right)+\rho K\right)=V\left(\operatorname{conv}\left(C_{n}\right)\right), V_{0}\left(\operatorname{conv}\left(C_{n}\right), K\right)=V(K)$, and $V_{i}\left(\operatorname{conv}\left(C_{n}\right), K\right)=0$ if $\operatorname{dim}\left(\operatorname{conv}\left(C_{n}\right)\right)<i$. Formula $(3.1)$ is an essential tool in the proof.

For a sausage configuration $S_{n}(u, K)=\left\{x^{1}, \ldots, x^{n}\right\}$ we have

$$
\operatorname{dim}\left(\operatorname{conv}\left(S_{n}(u, K)\right)\right)=1
$$

and thus

$$
V_{i}\left(\operatorname{conv}\left(S_{n}(u, K)\right), K\right)=0, \quad i=2, \ldots, d
$$

Moreover, since

$$
f_{\mathrm{DK}}\left(x^{i}-x^{i+1}\right)=2, \quad 1 \leq i \leq n-1,
$$

we get

$$
V_{1}\left(\operatorname{conv}\left(S_{n}(u, K)\right), K\right)=2(n-1)\left(f_{\mathrm{D} K}(u)\right)^{-1} V\left(K \mid u^{\perp}\right)
$$


where $K \mid u^{\curlywedge}$ denotes the orthogonal projection of $K$ onto the hyperplane with normal vector $u$ (see [Sc]). Hence

$$
V\left(\operatorname{conv}\left(S_{n}(u, K)\right)+\rho K\right)=2(n-1) \frac{V\left(K \mid u^{\perp}\right)}{f_{\mathrm{D} K}(u)} \rho^{d-1}+V(K) \rho^{d} .
$$

Proof of Theorem 1.2. Let $n \in \mathbb{N}, n>1, K \in \mathscr{K}^{d}, C_{n}=\left\{x^{1}, \ldots, x^{n}\right\} \in \mathscr{P}_{n}(K), \rho$ $\leq \frac{1}{32} d^{-1}(r(K) / R(K))$, and let $m=\operatorname{dim}\left(\operatorname{conv}\left(C_{n}\right)\right)$. To simplify the notation we write $R$ instead of $R(K)$. Let $z_{R} \in E^{d}$ with $K \subset z_{R}+R B^{d}$ and let $\bar{r}$ be the inradius of $\mathrm{D} K$. Then

$$
\bar{r} \geq r(K) .
$$

The proof now proceeds in four steps:

(1) We construct iteratively from $C_{n}$ distances $h_{j}$ and associated subspaces $L_{j}$ as follows: Without loss of generality let $x^{1}=0$ and let the diameter of conv $\left(C_{n}\right)$ be given by the distance of the points $x^{1}, x^{2}$. We set $h_{1}=\left|x^{1}-x^{2}\right| / 2$ and $L_{1}=$ $\operatorname{lin}\left\{x^{1}, x^{2}\right\}$, where $\operatorname{lin}(S)$ denotes the linear hull of a set $S$. Since $C_{n} \in \mathscr{P}_{n}(\mathrm{D} K)$ (see (1.1)) we have $h_{1} \geq \bar{r}$. Suppose we have chosen distances $h_{i}$ and linear spaces $L_{i}$, $1 \leq i<m$. Let $h_{i+1}=\max \left\{\operatorname{dist}\left(x^{k}, L_{i}\right): 1 \leq k \leq n\right\}$, where $\operatorname{dist}\left(x^{k}, L_{i}\right)$ denotes the Euclidean distance of $x^{k}$ to $L_{i}$. We may assume $h_{i+1}=\operatorname{dist}\left(x^{i+1}, L_{i}\right)$ and we set $L_{i+1}=\operatorname{lin}\left(L_{i} \cup\left\{x^{i+1}\right\}\right)$. We continue up to $i=m$ and obtain a monotone sequence of distances:

$$
2 h_{1} \geq h_{2} \geq h_{3} \geq \cdots \geq h_{m} \geq 0 .
$$

For $m=1$ we have $\operatorname{dim}\left(\operatorname{conv}\left(C_{n}\right)\right)=1$ and thus

$$
V\left(\operatorname{conv}\left(C_{n}\right)+\rho K\right) \geq V\left(\operatorname{conv}\left(S_{n}(K)\right)+\rho K\right) .
$$

So let $m>1, h_{m+1}=0$, and $l \in\{1, \ldots, m\}$ such that

$$
h_{l}>\frac{\bar{r}}{\sqrt{2}} \quad \text { and } \quad h_{l+1} \leq \frac{\bar{r}}{\sqrt{2}} .
$$

(2) For $1 \leq j \leq m$ let $C^{j}=L_{j} \cap \operatorname{conv}\left(C_{n}\right)$. Then $\operatorname{dim}\left(C^{j}\right)=j$ and we have, for the mixed volumes (see $[\mathrm{Sc}]$ ),

$$
\left(\begin{array}{l}
d \\
j
\end{array}\right) \rho^{d-j} V_{j}\left(\operatorname{conv}\left(C_{n}\right), K\right) \geq\left(\begin{array}{l}
d \\
j
\end{array}\right) \rho^{d-j} V_{j}\left(C^{j}, K\right)=\rho^{d-j} V\left(C^{j}\right) \cdot V\left(K \mid L_{j}^{\perp}\right) .
$$

Now let $v \in L_{j} \cap S^{d-1}$. Since $K \subset z_{R}+R B^{d}$ we have

$$
V\left(K \mid v^{\perp}\right) \leq \kappa_{j-1} R^{j-1} V\left(K \mid L_{j}^{\perp}\right)
$$

and so

$$
\left(\begin{array}{l}
d \\
j
\end{array}\right) \rho^{d-j} V_{j}\left(\operatorname{conv}\left(C_{n}\right), K\right) \geq \rho^{d-j} V\left(C^{j}\right) \cdot V\left(K \mid v^{\perp}\right) \kappa_{j-1}^{-1} R^{1-j}
$$


In the last two steps of the proof we consider the two cases $l=1$ and $l>1$.

(3) Let $l=1$. From (3.1), (3.2), and (3.4) we get, for $v \in L_{1} \cap S^{d-1}$,

$$
\begin{aligned}
& V\left(\operatorname{conv}\left(C_{n}\right)+\rho K\right)-V\left(\operatorname{conv}\left(S_{n}(v, K)\right)+\rho K\right) \\
& \quad \geq \sum_{j=1}^{2}\left(\begin{array}{c}
d \\
j
\end{array}\right) \rho^{d-j} V_{j}\left(\operatorname{conv}\left(C_{n}\right)\right)-\frac{2(n-1) \rho^{d-1} V\left(K \mid v^{\perp}\right)}{f_{\mathrm{D} K}(v)} \\
& \quad \geq \rho^{d-2} V\left(C^{2}\right) \cdot V\left(K \mid v^{\perp}\right) \frac{1}{2} R^{-1}+\rho^{d-1}\left(V\left(C^{1}\right)-\frac{2(n-1)}{f_{\mathrm{DK}}(v)}\right) V\left(K \mid v^{\perp}\right)
\end{aligned}
$$

Since $h_{j} \leq h_{2} \leq \bar{r} / \sqrt{2}$ for $j>1, \bar{r} B^{d} \subset \mathrm{D} K$, and $\left(1 / f_{\mathrm{D} K}(v)\right) B^{d} \cap L_{1} \subset \mathrm{D} K$, the intersection of $x^{i}+\mathrm{D} K$ with $L_{1}$ contains a line segment of length $2\left(1-h_{2} / \bar{r}\right) /$ $f_{\mathrm{DK}}(v), 1 \leq i \leq n$. Furthermore, since $C_{n}$ is a packing set with respect to $\mathrm{D} K$, any two of these segments have disjoint interiors and from the definition of $L_{1}$ we get $V\left(C^{1}\right) \geq 2(n-1)\left(1-h_{2} / \bar{r}\right) / f_{\mathrm{D} K}(v)$ and thus

$$
V\left(C^{2}\right) \geq V\left(\operatorname{conv}\left(C_{1} \cup\left\{x^{2}\right\}\right)\right) \geq h_{2}(n-1) \frac{1-h_{2} / \bar{r}}{f_{\mathrm{DK}}(v)} .
$$

Now $h_{2} / \bar{r} \leq 1 / \sqrt{2}$ and hence

$$
\begin{aligned}
& V\left(\operatorname{conv}\left(C_{n}\right)+\rho K\right)-V\left(\operatorname{conv}\left(S_{n}(v, K)\right)+\rho K\right) \\
& \quad \geq \rho^{d-2} \frac{2(n-1) V\left(K \mid v^{\perp}\right)}{f_{\mathrm{DK}}(v) \bar{r}} h_{2}\left(\frac{1}{4}\left(1-\frac{1}{\sqrt{2}}\right) \frac{\bar{r}}{R}-\rho\right) .
\end{aligned}
$$

By the choice of $\rho$ we obtain $V\left(\operatorname{conv}\left(C_{n}\right)+\rho K\right)-V\left(\operatorname{conv}\left(S_{n}(v, K)\right)+\rho K\right) \geq 0$.

(4) Let $l>1$. The construction of $C^{l}$ implies $V\left(C^{l}\right) \geq(2 / l !) \prod_{i=1}^{l} h_{i}$. Let $Q^{l}$ be the box with edge lengths $2 h_{1}, 2 h_{2}, \ldots, 2 h_{l}$ circumscribed to $C^{l}$ such that its facets have distance $h_{i}$ from $L_{i-1}, 2 \leq i \leq l$. Clearly,

$$
V\left(Q^{l}\right)=2^{l} \prod_{i=1}^{l} h_{i} \leq l ! 2^{l-1} V\left(C^{l}\right) .
$$

Let $\bar{x}^{j}, 1 \leq \mathrm{j} \leq n$, denote the projection of $x^{j}$ onto $L_{l}$ and let $v \in L_{l} \cap S^{d-1}$ be chosen such that $f_{\mathrm{DK}}(v)=\max \left\{f_{\mathrm{DK}}(u): u \in L_{l} \cap S^{d-1}\right\}$. Since $h_{l+1} \leq \bar{r} / \sqrt{2}$, the intersection of $x^{i}+\mathrm{D} K$ with $L_{l}$ contains an $l$-dimensional ball of radius $\gamma>0$, say, and center $\bar{x}^{i}, 1 \leq i \leq n$. Any two of these balls have disjoint interiors and are contained in a box with the same center as $Q^{l}$ and edge lengths $2\left(h_{1}+\gamma\right)$, $2\left(h_{2}+\gamma\right), \ldots, 2\left(h_{l}+\gamma\right)$. Thus $n \kappa_{l} \gamma^{l}<\Pi_{i=1}^{l}\left(1+\gamma / h_{i}\right) V\left(Q^{l}\right)$ or, with (3.5),

$$
2 n \kappa_{l} \leq V\left(C^{l}\right) l ! 2^{l} \cdot \prod_{i=1}^{l}\left(\frac{1}{\gamma}+\frac{1}{h_{i}}\right) .
$$


By the choice of $v$ we have

$$
\left(1 / f_{D K}(\nu)\right)\left(B^{d} \cap L_{l}\right) \subset \mathrm{D} K
$$

and hence $\gamma \geq\left(1-h_{l+1} / \bar{r}\right) / f_{\mathrm{DK}}(v)$. Together with $h_{1} \geq 1 / f_{\mathrm{D} K}(v)$ we obtain

$$
\frac{1}{\gamma}+\frac{1}{h_{1}} \leq \frac{2 \sqrt{2}-1}{\sqrt{2}-1} f_{\mathrm{DK}}(v)
$$

or

$$
\frac{2 n}{f_{\mathrm{DK}}(v)} \kappa_{l} \leq V\left(C^{l}\right) l ! 2^{l} \cdot\left(\frac{2 \sqrt{2}-1}{\sqrt{2}-1}\right) \prod_{i=2}^{l}\left(\frac{1}{\gamma}+\frac{1}{h_{i}}\right) .
$$

Since $\bar{r} B^{d} \subset \mathrm{D} K$ we also have $\gamma \geq\left(\bar{r}^{2}-h_{l+1}^{2}\right)^{1 / 2}$ and on account of $h_{i}>\bar{r} / \sqrt{2}$, $1 \leq i \leq l$, we get

$$
\begin{aligned}
\frac{2 n}{f_{\mathrm{DK}}(v)} \kappa_{l} & \leq V\left(C^{l}\right) l ! 2^{l} \cdot\left(\frac{2 \sqrt{2}-1}{\sqrt{2}-1}\right)(2 \sqrt{2})^{l-1} \bar{r}^{1-l} \\
& =V\left(C^{l}\right) l ! 2^{(5 l-3) / 2} \cdot\left(\frac{2 \sqrt{2}-1}{\sqrt{2}-1}\right) .
\end{aligned}
$$

Now we use this lower bound of $V\left(C^{l}\right)$ in formula (3.4) and by (3.1)-(3.3) and the choice of $\rho$ we find

$$
\begin{aligned}
& V\left(\operatorname{conv}\left(C_{n}\right)+\rho K\right)-V\left(\operatorname{conv}\left(S_{n}(v, K)\right)+\rho K\right) \\
& \geq \frac{2(n-1) V\left(K \mid v^{\perp}\right)}{f_{\mathrm{DK}}(v)} \rho^{d-l}\left(\frac{r(K)}{R}\right)^{l-1} \\
& \quad \times\left(\frac{\kappa_{l}}{\kappa_{l-1}}(l !)^{-1} 2^{(3-5 l) / 2}\left(\frac{2 \sqrt{2}-1}{\sqrt{2}-1}\right)^{-1}-(32 d)^{1-l}\right) .
\end{aligned}
$$

Let the term in the last brackets be denoted by $g(l, d)$. It remains to show that $g(l, d) \geq 0$ for $2 \leq l \leq d$. Obviously, $g(l, d) \geq g(l, l)$ and for $l=2$ it can easily be checked that $g(l, l)>0$. So let $l \geq 3$. With $(2 \sqrt{2}-1) /(\sqrt{2}-1)<\frac{9}{2}, \kappa_{l} / \kappa_{l-1}>$ $(2 \pi /(l+1))^{1 / 2}[\mathrm{BGW}]$ and Stirling's formula $l !<(l / e)^{i}(2 \pi(l+1))^{1 / 2}$ we get

$$
g(l, d) \geq g(l, l)>\left(\frac{1}{l} \frac{e}{4 \sqrt{2}}\right)^{l-1}\left(\frac{e}{9(l+1) l}-\left(\frac{\sqrt{2}}{8 e}\right)^{l-1}\right) .
$$

From this the assertion follows, since $h(l):=(l(l+1))^{-1 /(l-1)}$ is monotonely increasing and the difference in the large brackets is positive for $l=3$. 
Let us remark that the constant factor in the upper bound of $\rho$ can easily be improved, but clearly this factor has no influence on the asymptotic behavior of the bound as $d$ tends to infinity.

\section{References}

[BGW] U. Betke, P. Gritzmann, and J. M. Wills, Slices of L. Fejes Tóth's sausage conjecture, Mathmatika 29 (1982), 194-201.

[BHW] J. Betke, M. Henk, and J. M. Wills, Finite and infinite packings. J. Reine Angew. Math. 453 (1994), 165-191.

[Bl] H. F. Blichfeldt, The minimum value of quadratic forms, and the closest packings of spheres, Math. Ann. 101 (1929), 605-608.

[CS] J. H. Conway and N. J. A. Sloane, Sphere Packings, Lattices and Groups, 2nd edn., Springer-Verlag New York, 1993.

[Fe] L. Fejes Tóth, Research problem 13, Period. Math. Hungar. 6 (1975), 197-199.

[FK] G. Fejes Tóth and W. Kuperberg, Packing and covering with convex sets, Handbook of Convex Geometry, Vol. B (P. M. Gruber and J. M. Wills, eds.), North-Holland, Amsterdam, 1993, pp. 799-860.

[GL] P. M. Gruber and C. G. Lekkerkerker, Geometry of Numbers, 2nd edn., North-Holland, Amsterdam, 1987.

[Gr1] H. Groemer, Über die Einlagerungen von Kreisen in einem konvexen Bereich, Math. Z. 73 (1960), 285-294.

[Gr2] H. Groemer, Existenzsätze für Lagerungen im Euklidischen Raum, Math. Z. 81 (1963), 260-278.

[GW] P. Gritzmann and J. M. Wills, Finite packing and covering, Handbook of Convex Geometry, Vol. B (P. M. Gruber and J. M. Wills, eds.), North-Holland, Amsterdam, 1993, pp. 861-897.

[Jo] F. John, Extremum problems with inequalities as subsidiary conditions, Studies and Essays, Courant Anniversary Volume, Interscience, New York, 1948, pp. 187-204.

[KK] D. J. Kleitman and M. K. Krieger, An optimal bound for two-dimensional bin packing, Proc. 16th Ann. Symp. on Foundations of Computer Science, 1975, pp. 163-168.

[KL] G. A. Kabatjanskili and Levenštein, Bounds for packings on a sphere and in space, Problemy Peredachi Informatsii 14 (1978), 3-25 (Russian), English transl., Problems Inform. Transmission 14 (1978), 1-17.

[Ol] N. Oler, An inequality in the geometry of numbers, Acta Math. A (1961), no. 105, 19-48.

[Ro1] C. A. Rogers, The closest packing of convex two-dimensional domains, Acta Math. 86 (1951), 309-321.

[Ro2] C. A. Rogers, Packing and Covering, Cambridge University Press, Cambridge, 1964.

[Sc] R. Schneider, Convex Geometry: The Brunn-Minkowski Theory, Cambridge University Press, Cambridge, 1992.

[Th] A. Thue, Über die dichteste Zusammenstellung von kongruenten Kreisen in einer Ebene, Kra. Vidensk. Selsk. Skrifter, I. Math. Nat. Kl. 1910 (1910), 257-263.

Received January 15, 1994. 